Objectives: To evaluate the effects of the disease and it's treatment on serum $\mathrm{AMH}$ levels for Behcet's disease (BD), systemic lupus erythematosus (SLE), rheumatoid arthritis (RA), and systemic scleroderma (SSD).

Methods: The study included 73 patients with RDs from 18 to 40 years: 42 patients with BD, 12 with SLE, 11 with RA, 8- with SSD, the control group consisted of 15 healthy women. Enzyme-linked immunosorbent assay (ELISA) was used to measure AMH levels. Parametric and nonparametric statistical methods of Statistica 8.0 package were used for statistical processing of data.

Results: Mean age in BD patients was 30.0 years, in SLE and RA -33.5 years, in SSD - 35.0 years, and 32.0 years in the control group. The average disease duration was 4.5 years, 11.5 years, 4.0 years and 6.0 years, respectively.

\begin{tabular}{lccccc}
\hline \multicolumn{7}{c}{$\mathrm{BD}, \mathrm{n}=42$} & $\mathrm{SLE}, \mathrm{n}=12$ & $\mathrm{RA}, \mathrm{n}=11$ & $\mathrm{SSD}, \mathrm{n}=8$ & Control, $\mathrm{n}=15$ \\
\hline Mean age, years & 30,0 & 33.5 & 33,5 & 35,0 & 32,0 \\
& {$[26 ; 35]$} & {$[29 ; 38]$} & {$[24 ; 36]$} & {$[28 ; 40]$} & {$[26 ; 35]$} \\
Average disease duration, years & 4,5 & 11,5 & 4,0 & 6,0 & - \\
& {$[2,6 ; 9,0]$} & {$[2,8 ; 18]$} & {$[4,0 ; 6,0]$} & {$[2,0-10,0]$} & \\
Mean AMH level, ng/ml & 2,5 & 3,5 & 3,35 & $2,5[0,3 ; 7,1]$ & 3,1 \\
& {$[1,1 ; 3,7]$} & {$[0,4-7,1]$} & {$[2,0 ; 7,6]$} & & {$[1,9 ; 5,4]$}
\end{tabular}

There were no significant differences in the mean AMH levels between the groups. No association between AMG levels and clinical manifestations, disease activity or duration of rheumatic disease was found. Baseline $\mathrm{AMH}$ - in treatment-naïve patients before initiation of any DMARDs was assessed in 11 BD patients. A significant $(p>0.05)$ decrease of AMH levels was established in patients with high SLE activity treated with CP. Of notice, all examined patients were additionally receiving a GEBD -Rituximab.

Conclusion: Decreased ovarian function was found in patients with high SLE activity treated with CP with Rituximab.

Disclosure of Interests: None declared

DOI: 10.1136/annrheumdis-2020-eular.3465

\section{AB0478 CORONARY ATHEROSCLEROSIS IN BEHCET'S DISEASE}

R. Goloeva', Z. Alekberova'. ' V.A. Nasonova Research Institute of

Rheumatology, Moscow, Russian Federation

Background: Behcet's disease (BD) is systemic vasculitis, which affects all types and sizes of vessels. Increased carotid intima-media thickness (IMT) is parameter associated with subclinical atherosclerosis.

Objectives: To determine the prevalence of atherosclerosis in pts with $\mathrm{BD}$.

Methods: 95 BD pts were evaluated and 45 healthy controls matched for age and gender.

IMT was assessed by high-resolution B-mode ultrasonography. Serum concentration of high-sensitivity $\mathrm{C}$-reactive protein (hs $\mathrm{CRP}$ ) was measured by immunonephelometric assay (BN-100 Analyzer; Dade Behring). Lipid profile evaluation included total cholesterol, TGs, HDL, LDL and atherogenic index.

Results: The male-to-female ratio was 3,7:1, the mean age of pts was 29.7 (23$35) \mathrm{yrs}$, the mean age at the disease onset - 19,9 (14-25) yrs, the mean disease duration - 9,6 (4-15) yrs.

\begin{tabular}{lccc}
\hline & BD, \% & Healthy, \% & $\mathrm{P}$ \\
\hline Arterial hypertension & 22,1 & 13,3 & $\mathrm{~ns}$ \\
Smoked & 29,5 & 24,4 & $\mathrm{~ns}$ \\
Diabetes mellitus & 0 & 0 & $\mathrm{~ns}$ \\
Lipid abnormalities & 76,8 & 64,4 & $\mathrm{~ns}$ \\
Total cholesterol & $5,0 \pm 1,40$ & $5,3 \pm 0,96$ & $\mathrm{~ns}$ \\
TGs & $0,91 \pm 0,65$ & $0,90 \pm 0,68$ & $\mathrm{~ns}$ \\
HDL & $1,18 \pm 0,39$ & $1,26 \pm 0,29$ & $\mathrm{~ns}$ \\
LDL & $3,40 \pm 1,46$ & $3,47 \pm 0,90$ & $\mathrm{~ns}$ \\
Hs CRP & $2,42 \pm 21,0$ & $0,3 \pm 0,63$ & $<0,00$ \\
Mean IMT carotid artery & $0,68 \pm 0,09$ & $0,64 \pm 0,09$ & $\mathrm{~ns}$ \\
IMT $<0,5$ mm & 31,6 & 6,6 & $<0,00$ \\
IMT $>0,5-0,89$ mm & 49,5 & 86,6 & $\mathrm{~ns}$ \\
IMT - 0,9-1,2 mm & 13,68 & 6,6 & $\mathrm{~ns}$ \\
IMT $>1,2$ mm (plaques) & 5,7 & 0 & $\mathrm{~ns}$ \\
\end{tabular}

The mean carotid IMT in BD pts was similar with controls. In pts thinning IMT and concentration of hsCRP was significantly higher compared with controls.

IMT correlated with age of pts, concentration of total cholesterol, LDL and hsCRP but did not correlated with clinical manifestation and disease duration. Carotid atherosclerotic plaques were found in 5 pts with BD - mean age $40,0 \pm 8,1$, who had traditional cardiovascular risk factors (high body masse index, hypertension) and received long time therapy with glucocorticoids.
Conclusion: Coronary atherosclerosis in BD pts was lower than what we expected. The thinning IMT may be one of the risk factors for aneurysm formation in pts with BD.

Disclosure of Interests: None declared

DOI: 10.1136/annrheumdis-2020-eular.3609

\section{AB0479 PREGNANCY OUTCOMES IN PATIENTS WITH RHEUMATIC DISEASES.}

Z. Alekberova ${ }^{1}$, R. Goloeva ${ }^{1}$, A. Lila ${ }^{1}$. ${ }^{1}$ V.A. Nasonova Research Institute of Rheumatology, Moscow, Russian Federation

\section{Background:}

Objectives: To evaluate pregnancy outcomes in patients with Behcet's disease (BD), systemic lupus erythematosus (SLE), rheumatoid arthritis (RA), and systemic scleroderma (SSD).

Methods: The study included 73 patients with rheumatic diseases (RDs) aged 18 to 40 years signing the informed consent form and completing dedicated thematic chart. The mean age of participants was 30.0 years - for BD patients; 33.5 years - for SLE, 33.5 - for RA; and 35.0 for SSD patients; mean disease duration was 4.5 years 11.5 years 4.0 years and 6.0 years, respectively. 23 patients (11 BD patients, 5 SLE, 5 RA 5 and 2 SSD 2) out of 73 never had pregnancies, including 15 virgo patients and 4 patients who were planning pregnancy in the near future. 158 pregnancies in 50 patients with rheumatic diseases (31 BD patients, 7 SLE, 6 RA, and 6 SSD pts) were retrospectively analyzed.

Results: Outcomes of 159 pregnancies in RD patients were as follows: 95 (60\%) on time births of full-term babies; 2 cases (1.2\%) of early neonatal death in two $\mathrm{BD}$ females (premature deliveries at 28 and 32 gestational weeks of babies with signs of extreme prematurity who died 1 day after birth); 35 pregnancies were terminated before 10 weeks of gestation: 34 pregnancies (in 4 patients) - at the woman's request, 1-for medical reasons (rubella at 7 weeks of gestation).

Totally 27 cases of adverse pregnancy outcomes were documented in 20 patients ( $40 \%$ of 50 patients): 19 cases (in 14 patients) of intrauterine deaths, one case of ectopic pregnancy, 8 cases (in 6 patients) of early miscarriages (at 5,6 and 10 weeks of gestation).

There were no cases of antiphospholipid syndrome documented.

Pregnancy outcomes in RD patients

\begin{tabular}{lcccc}
\hline & BD, $\mathrm{n}=31$ & SLE, $\mathrm{n}=7$ & $\mathrm{RA}, \mathrm{n}=6$ & SSD, $\mathrm{n}=6$ \\
\hline Pregnancy, $\mathrm{n}$ & 107 & 16 & 17 & 18 \\
Childbirth, $\mathrm{n}$ & 71 & 9 & 9 & 6 \\
Medical abortion, $\mathrm{n}$ & 20 & 2 & 3 & 10 \\
Intrauterine deaths, $\mathrm{n}$ & 12 & 4 & 4 & 0 \\
Miscarriage, $\mathrm{n}$ & 4 & 1 & 1 & 2 \\
\hline
\end{tabular}

Out of total 95 babies born to RD patients, two had recurrent aphthous stomatitis (in $2 \mathrm{BD}$ patients), and one had congenital glaucoma (born to a mother with neuro-Behcet's).

$10 \mathrm{BD}$ and $2 \mathrm{RA}$ are patients multipara mothers who are raising 3 and more children.

Conclusion: Rheumatic diseases are associated with high incidence of adverse pregnancy outcomes.

Disclosure of Interests: None declared

DOI: 10.1136/annrheumdis-2020-eular.3704

\begin{tabular}{|l|l}
\hline AB0480 & AN OPEN-LABEL, EXPLORATORY STUDY TO \\
ESTABLISH THE EFFICACY AND SAFETY OF 1-YEAR \\
CANAKINUMAB TREATMENT IN BEHÇET'S DISEASE \\
PATIENTS WITH NEUROLOGIC OR VASCULAR \\
INVOLVEMENT
\end{tabular}

A. Gül' ${ }^{1}$, M. Kurtuncu ${ }^{1}$, M. Erdugan ${ }^{1}$, E. Oguz ${ }^{2}$, T. Gunduz ${ }^{2}$, G. Akman Demir ${ }^{2}$, S. Sevgi ${ }^{3}$, S. Turgay ${ }^{4}$, E. Acar ${ }^{5} .{ }^{1}$ Istanbul Faculty of Medicine, Istanbul, Turkey; ${ }^{2}$ Istanbul Faculty of Medicine, Istanbul, Turkey; ${ }^{3}$ Novartis Turkey, Istanbul, Turkey; ${ }^{4}$ Novartis Turkey, Istanbul, Turkey; ${ }^{5}$ Novartis Turkey, Istanbul, Turkey

Background: Previous observations in Behcet's disease (BD) patients receiving anti-IL 1 therapies suggested that dosage and route of administration may be critical in controlling disease manifestations. This was supported by favorable observations with infliximab infusions reaching higher serum trough levels in refractory BD patients compared to other anti-TNF agents.

Objectives: The primary objective was to evaluate the safety and efficacy of intravenous (IV) canakinumab (CAN) on the clinical and inflammatory findings of $\mathrm{BD}$ patients with neurologic and vascular involvement.

Methods: Biologic-naïve BD patients, who had a recent attack of large vessel vascular or parenchymal neurologic disease within the last month were enrolled and all received $300 \mathrm{mg}$ CAN IV without a change in other medications. Response was assessed on day 30 as the primary objective; partial 